\title{
SERGEY DAVYDOV (ED.) (2020). INTERNET IN RUSSIA. A STUDY OF THE RUNET AND ITS IMPACT ON SOCIAL LIFE. CHAM: SPRINGER NATURE SWITZERLAND AG, 298 PP., ISBN 978-3-030-33015-6.
}

Internet in Russia. A Study of the Runet and Its Impact on Social Life edited by Sergey Davydov was one of the most interesting promises expected in 2020 and published by Springer Nature Switzerland AG. For a researcher of communication and media, the title seemed to be intriguing at least for two reasons. The editor explains the main idea of the book in the opening sentences of the publication:

"What is Runet? There are at least three possible answers to this question. First, it is a segment of the Internet with content in Russian language. Second, it is a segment of the Internet associated with the domain zone.RU. Third, it is the national-Russian-segment of the Internet. The latter definition is the closest one to the idea of this book".

Firstly, it is a bold thesis, especially because this way of thinking about online reality is often criticized by network researchers. As we know, the internet differs from other types of media and communication tools by not having its own nationality. The network embraces the whole world, and its language mutations or those based on national domains seem insignificant from a global perspective (according to W3Techs, websites in Russian include $8.6 \%$ of online resources in 2020). Secondly, this way of thinking about your own uniqueness may be the source of the original sin: seeing specificity in something that ultimately turns out to be universal. Internet in Russia - by the title itself - tried to contradict these paradigms.

The book consists of an introduction and four parts (Theory and History, Economy and Regulation, Digital Culture, Participation, Representations and Discussions). Among them, 15 articles and the Afterwords can be found. A fairly large number of articles for a composite work have undoubtedly something in common - meticulous source queries made by all of the authors. It results in a solidly prepared theoretical basis for the reflections presented in the book and seems to be one of the greatest advantages of this work. Anyone who would like to deal with the local contexts of how the internet functions should treat this publication as a kind of textbook, in which one can find cataloged and richly described media/communication theories. All the articles also include the long lists of sources that may enrich the reader's knowledge about internet research. 
On this basis, the book constructs a broad and detailed picture of the internet in Russia. Numerous perspectives can be found - historical, media studies, legal, etc., which proves the various points of view of the authors invited to publish articles. This fact makes the book full of interesting paradigms for a researcher of the internet sphere. However, in such an editorial solution there is a threat to the reader: surely not all perspectives will be equally interesting for everyone. Additionally, the texts are quite unequal in terms of research value. So perhaps it would have been worthwhile to have employed the old marketing adage: less is more.

The variety of threads is overwhelming, which makes the book a complex "science fabric". Unfortunately, there are a lot of dead ends: strands related to media other than the internet can be found, which go beyond the declaration made in the title. But also, for example, out-of-date data appears (see below), which is an unforgivable sin in this type of publication. The internet, so dynamically changing, requires special care as a subject of knowledge. The date of publication of the book should coincide with the current data taken from reliable sources. Authors of the articles are not consistent in applying this rule.

Reading consecutives chapters, there is the impression that the division of the chapters seems to be dictated by the subject matter of individual interests of the researchers invited to the publication. It always should be made the other way round - the chapter content is adjusted to the general concept of the book. As a result, both the list of topics, as well as the division into individual chapters, seems to be rather random. This is quite a common problem in such books, especially when including a long list of publications. The inability to thoroughly discuss the concept of a book with all the researchers has the tendency to result in a mosaic of texts that do not always correspond to one another. Accordingly Internet in Russia... does not seem to be the conceptually coherent text. The most glaring and banal proofs of this are uneven technical issues - e.g., various fonts in graphics, which are sometimes blurred and underdeveloped (p. 108). The proof from the content can be found in the large inconsistency of the texts.

Although individual texts have well-structured compendiums on theories and concepts devoted to internet research, the book as a whole does not contribute much to scientific knowledge in the analyzed area. Therefore, it is worthwhile giving some attention to the 15 articles published in this publication.

The opening text (Digital Inequalities in European Post-Soviet States) is a comparison of the development of the internet in post-Soviet countries, showing the historical dimension of the problem. However, it partly contains data until 2016, which makes it difficult to consider its conclusions as up-to-date. Similar problems can be found in the other article: Elite Russian Students' Internet Strategies: Trust, Persuasion, and Rejection, in which conclusions are partly based on research conducted in 2011, i.e., a decade ago. 
Many interesting statistical data are also provided by the author of the second text (The Internet in the Structure of the Russian Media System). However, a lot of side threads (e.g., regarding other types of media) and modest conclusions based on them give the impression of "distorted proportions" and cause a lack of satisfaction at the analytical field, leaving the reader with a descriptive image of online reality. A similar problem concerns the text Runet in Crisis Situations. The data collected and used in the text seems to be not sufficiently analyzed and not backed up by own research.

The third text (The Rise of Runet and the Main Stages of Its History) is an example of the above-mentioned inconsistency throughout the idea of the book. One of the authors claims that the history of the internet in Russia has already been described. Meanwhile, another text on the same subject was created as part of the publication. As in the previous text, a lack of conclusions and a shortage of more cross-sectional analytical thought is visible. The same problem applies to the following two chapters (Investments in Runet; Regulation of Online Freedom of Expression in Russia in the Context of the Council of Europe Standards), in which a lot of data does not translate into an in-depth analysis. The latter of the two chapters is also based on questionable methodology.

The sixth contribution (Digital Literacy Concepts and Measurement) takes up an important problem, recalling various studies in an interesting way, but without in-depth statistical analysis that could be a key enrichment of it (taking into account e.g., criteria such as age, education, economic status, etc.). Another article (Journalistic Cultures: New Times, New Gaps?) is also cognitively valuable, but the author falls into the trap of an excess of data and gives the impression that it does not fit the concept and the main goals of the publication formulated in the introduction.

The next article (Diversity of the Internet in Russia's Regions: Towards an Alternative Research Agenda) is the case study of several regions, but with very vague criteria for selecting cases and for the conducted analysis. A similar problem accompanies the research presented in the other text (Russia in International Social Media Discussions: Pro and Contra). The author writes: "The relevance and importance of the communities and groups were decided on the basis of the size of the membership (looking for higher membership), the frequency of postings and quality and quantity of the resulting discussion". It is difficult to find here a clear criterion for selecting a research sample.

The same doubt can be found in the article Making Ruins Great Again: Documentation and Participation on Instagram. This text additionally shows another problem: quoting a few comments without any clear criteria, a codebook, operationalized concepts, a shared database does not render the research into a content analysis, as the author declares. It may be concluded that the subjective 
approach to the above-mentioned issues basically overturns the scientific value of such an analysis.

The text Data Turn and Datascape in Russia is the most symptomatic of the entire volume, clearly reflecting its problems. One article devoted to smart cities, medicine, education, art, etc. makes it difficult to follow the author's line of thought. At the same time - as in the entire publication - there are no threads related to the threats arising from the described and studied trends and phenomena. These are common in scientific dialogue and are beginning to dominate the literature on internet issues. It should be also admitted that this text, one of the few, tackles contemporary problems generated by the network (artificial intelligence, big data, etc.) that are important from the point of view of researchers all over the world.

Undoubtably crucial topics accompany articles on progressive and left-wing movements: The internet: Its Influences on Environmental Communication and Environmental Movements as well as Gender Activism in the Russian Segment of the Internet. However, it is also difficult to find the author's perspective of research in either of the texts. Additionally - in the latter text - reducing the \#metoo phenomenon to a flashmob seems to be at the least a controversial procedure.

It seems that Afterwords, by Marlene Laruelle, outlined the problems of the internet in contemporary Russia in the most interesting way. It would be worth following her diagnoses on this issue if the book project were to have any continuation. So perhaps the distanced perspective of the outside observer is more proper when trying to describe a mediated area anchored in a specific cultural area. It is well known that we cannot build a vision of identity without comparing it to others (actually only one author of the opening text does it, and to a limited extent). So maybe other countries are also facing similar problems, but after reading Davydov's book we will not be able to find it out. Is it possible that the qualities of Runet, ipso facto, are not specific only to Russia?

The book, published by Springer, is a good starting point for those who are looking for basic knowledge about internet research from a territory-oriented approach. The impressive list of theories and concepts in which the researchers have embedded their thoughts is worth emphasizing. However, this should not divert attention from errors in the research procedures and shortcomings in the individual parts of this work. Perhaps the best symbol of this is the cover of the publication - a graphic symbolizing the fall of the Berlin Wall announcing a book about contemporary Russia. Something is wrong here.

Jakub Jakubowski ADAM MICKIEWICZ UNIVERSITY IN POZNAŃ, POLAND 